\title{
SIMPLE analytical model for smart microfluidic chip design
}

\author{
Francesco Dal Dosso a,1, Yura Bondarenko a,b,1, Tadej Kokalj,a and Jeroen Lammertyn a* \\ a Department of Biosystems, MeBioS - Biosensors Group, KU Leuven, 3001 Leuven, Belgium \\ b Department of Biosystems, MeBioS - Biophotonics Group, KU Leuven, 3001 Leuven, Belgium \\ 1 These authors contributed equally to this work. \\ * Corresponding author: jeroen.lammertyn@kuleuven.be
}

\begin{abstract}
Precise control of the flow dynamics in a microfluidic device is of great importance for the integration of bioassays on-chip. Recently, the Self-powered Imbibing Microfluidic Pump by Liquid Encapsulation(SIMPLE) was developed in our group and integrated with biological applications. The system functions based on capillary imbibition of a working liquid (WL) into a porous material (PM), which in turn pulls a sample liquid (SL) through the connected microfluidic channel network. Analytical models describing the pumping dynamics of paper-based and channel-based systems have been presented, but no suitable analytical models have been reported for hybrid systems such as SIMPLE. Moreover, the available models were mostly limited to only describing the pumping process (i.e. flow rate) for given design parameters (i.e. paper shape, channels geometry), which still resulted in tedious trial-and-error process to optimize the chip design to achieve the desired flow rate. In this work, we developed a smart designing tool for SIMPLE-based chips that provides the design parameters necessary to obtain a targeted flow rate. An analytical model for the SIMPLE was first derived and validated, confirming its 3 main hypotheses: i) the sample flow rate is dependent on the porous material geometry but independent from the ii) porous material volume and iii) channel geometry. All experimental results were in good agreement with this model. Finally, we used our model as a prediction tool providing precise design parameters to avoid the time-consuming trialand-error approach needed to achieve a specific flow rate. In particular, several chips were fabricated according to the model inputs and the sample liquid flow rates measured $(1.5 \pm 0.3,5.3 \pm 1.5,15.2 \pm$ $2.7 \mu \mathrm{L} / \mathrm{min}$ ) were matching the targeted ones $(1.5,5,15 \mu \mathrm{L} / \mathrm{min})$. The analytical model developed in this work was proven to be a useful designing tool for fast and efficient optimization of SIMPLE-based chips in order to address specific application requirements.
\end{abstract}

\section{Keywords:}

Self-powered microfluidics; analytical model; flow-rate design tool; SIMPLE pump

\section{Introduction}

Passive, self-powered micropumps are ideal for portable lab-on-a-chip (LOC) devices, especially for point-of-care (POC) applications [1], since they eliminate the need for external actuators or power sources. Different approaches to self-powered microfluidics have been presented and surface tension-based pumping platforms are the most investigated. Here, pumping relies on liquid wicking by capillary action into hydrophilic capillaries, micropost structures or porous materials (i.e. paper) [2-11]. Even though these approaches have several advantages (i.e. self-powered, portability, 
robustness, affordability), they suffer from specific limitations. In self-powered channel-based systems, channels need to be hydrophilized to obtain the capillary effect with time-consuming and skill-demanding treatments, while in paper-based concepts, the transport of analytes through the matrix can decrease the sensitivity and specificity of the analysis due to interferences.

To overcome this, the benefits of paper-based and channel-based microfluidics have been combined in so-called hybrid microfluidic devices. In fact, these approaches take advantage of the capillary wicking in paper substrates to transport liquids in void microfluidic channels. Only a few approaches in this field have been published [7,12-14] which still present some drawbacks such as the need of hydrophilic channels and the impossibility to work with small sample volumes. These issues were solved in the self-powered imbibing microfluidic pump by liquid encapsulation (SIMPLE) presented by Kokalj et al. [15] where a prefilled liquid and a porous material form an integrated capillary pump that pulls another liquid (i.e. sample) into a microfluidic network. Importantly, with this system the sample does not get in contact with the porous material itself, avoiding the interference problem typical for traditional paper-based systems.

However, all the aforementioned approaches present a common issue: the flow control of pumping systems are generally fixed by the design and cannot be adjusted after the chip fabrication, which limits the flexibility and increases the chip optimization time. Because a precise control of the flow dynamics in a microfluidic chip is of great importance for the development of POC bioassays, the chip needs to be carefully designed upfront in order to provide adequate performance for the targeted application [9,16]. For instance, in paper-based microfluidics, the basic method of controlling liquid flows is by changing the type $[17,18]$ or shape (i.e. circular, rectangular, sectorshapes) [19-23] of the porous material. However, in these papers, the flow rate is first measured for a specific design and if it needs to be adjusted, the chip design is modified in an iterative way until the desired flow rate is obtained. In other studies, an reverse calculation approach was developed to predict upfront the exact design that provided the specific flow rate $[24,25]$. This gave more insight in the flow dynamics of these systems and reduced the optimization time significantly. However, this inverted approach has not been described for hybrid systems yet, and in particular the mechanisms behind liquid transport in the SIMPLE platform have not been studied in depth. Until now, SIMPLE chips were designed based on an inefficient trial-and-error approach prone to variability.

Since a precise understanding of the flow dynamics in a microfluidic chip is of great importance for the development of POC bioassays and the SIMPLE technology has proven to have the potential for being an ideal POC platform [26], we present here an analytical model to describe the pumping behavior of the SIMPLE. This model combines different equations of either paper- or channel-based systems in order to describe the SIMPLE hybrid approach. First, the analytical derivation of the model is shortly presented. Then, the model is validated experimentally for both different porous material properties (i.e. size and shape) and channel dimensions. Finally, the model is used as a smart design tool: different chips are designed to achieve desired flow rates and the predicted values are compared to the experimental measurements, showing great agreement. 


\section{Analytical derivation of the SIMPLE flow dynamics}

\subsection{SIMPLE platform description}

A SIMPLE chip consists of a porous material (PM), a working liquid channel (WLC) and a sample liquid channel (SLC), as illustrated in Figure 1. To operate a SIMPLE chip, a working liquid (WL) is first prefilled in the WLC and a droplet of sample liquid (SL) is deposited on the inlet. By applying a small pressure on the WLC, the WL contacts the PM and gets absorbed. The absorption of the WL into the PM creates an under pressure in the SLC which draws the SL into the microfluidic network [15].

The geometrical parameters of a SIMPLE chip are defined as given in Figure 1. In particular, $w_{w}[\mathrm{~m}]$ and $w_{s}[\mathrm{~m}]$ represent the WLC and SLC width, respectively, $l_{0}[\mathrm{~m}]$ the WLC length and $h[\mathrm{~m}]$ defines the height of the microfluidic channels. The PM is geometrically defined by its central angle $\beta[\mathrm{rad}]$, its thickness $h_{p}[\mathrm{~m}]$ and the initial radius $r_{0}[\mathrm{~m}]$. Additionally, a number of parameters are required to represent the properties of the PM, such as the porosity $\phi[-]$, the pore radius $r_{p}[m]$ and the permeability $\kappa\left[\mathrm{m}^{2}\right]$. The dynamic state of the SIMPLE pump is described by its imbibition radius $r[m]$, WLC length $\ell_{w}[m]$ and SLC length $\ell_{s}[m]$, all three in function of time $t[s]$.

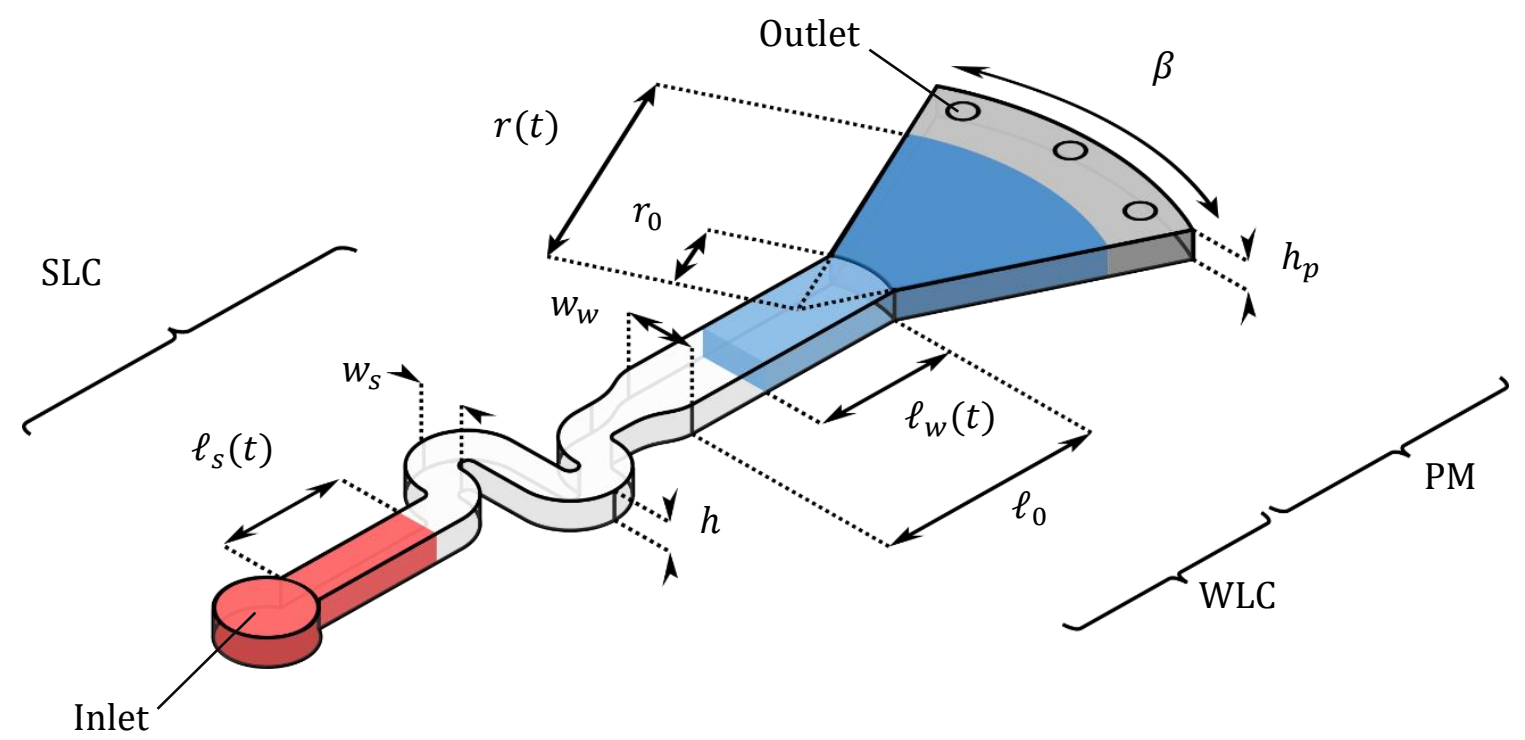

Figure 1: Schematic representation of a SIMPLE in the pumping regime. Three main parts can be identified: the PM, the WLC with prefilled WL (blue liquid) and the SLC with SL (red liquid). The chip inlet is at the beginning of the SLC while the outlet is made of vent holes at the end of the PM. The dynamics of the pumping process are described by the imbibition radius $(\boldsymbol{r})$ and the position of the menisci in the WLC and SLC $\left(\boldsymbol{\ell}_{\boldsymbol{w}}\right.$ and $\left.\boldsymbol{\ell}_{\boldsymbol{s}}\right)$ in function of time. These variables can be expressed in terms of the design parameters $\left(\boldsymbol{\beta}, \boldsymbol{r}_{\mathbf{0}}, \boldsymbol{h}, \boldsymbol{w}_{\boldsymbol{w}}, \boldsymbol{w}_{\boldsymbol{s}}, \boldsymbol{\ell}_{\mathbf{0}}\right)$ and the PM properties $\left(\boldsymbol{\phi}, \boldsymbol{r}_{\boldsymbol{p}}, \boldsymbol{\kappa}, \boldsymbol{h}_{\boldsymbol{p}}\right)$.

\subsection{SIMPLE governing equations and hypotheses}

After the chip activation when an external pressure is applied (i.e. fingertip pressure), during the pumping process, there is no pressure drop between the inlet and the outlet of the chip. However, pressure differences arise in the chip itself: the capillary pressure $\Delta p_{c a p}[\mathrm{~Pa}]$ at the imbibition front, $\Delta p_{\text {cap }}$ between the imbibition front and the PM-WLC interface, and $\Delta_{\text {chan }}$ between the PM-WLC interface and the inlet of the SLC. As the WL wicks into the paper, the pressure drop over the PM increases. Simultaneously, the WLC is depleted of WL while SL starts to flow into the SLC, 
continuously changing the hydraulic resistance of the channels which also depends on the length of the filled channel.

Given the relative large (for microfluidic standard) channel dimensions $\left(0.5 \mathrm{~mm}<w_{w}, w_{s}<\right.$ $2 \mathrm{~mm}$ and $h=0.153 \mathrm{~mm}$ ) and the relatively slow flow rates (in the range of $\mu \mathrm{L} / \mathrm{min}$ ) achieved in the SIMPLE system [15,26,27], inertial effects as well as geometrical features such as bends in the channels can be neglected $(R e<1)$. Assuming no significant compressive or inertial effects are present as discussed in Section A.1 of the Appendix, the total volume of SL and WL in the channels is directly related to the volume of WL absorbed in the PM. Considering this, the pressure balance can be formulated as shown in Equation (1), with all terms expressed in function of $r$ (Equations (2) through (4)). By substituting Equations (2) through (4) in Equation (1) a first-order differential Equation (5) is obtained:

$$
\begin{aligned}
& \Delta p_{\text {cap }}=\Delta p_{\text {por }}+\Delta p_{\text {chan }} \\
& \text { Young-Laplace } \\
& \Delta p_{\text {cap }}=-\frac{2 \gamma \cdot \cos \theta_{p}}{r_{p}} \\
& \text { Darcy's Law } \quad \Delta p_{\text {por }}=\frac{\mu \phi}{\kappa} \cdot r \ln \left(\frac{r}{r_{0}}\right) \frac{d r}{d t} \\
& \text { Hagen-Poiseuille } \quad \Delta p_{\text {chan }}=\mu\left[R_{W}(r)+R_{S}(r)\right] \cdot\left|\phi \beta h_{p} r \frac{d r}{d t}\right| \\
& \left(\alpha \ln \left(\frac{r}{r_{0}}\right)+C_{1}\left(r^{2}-r_{0}^{2}\right)+C_{2}\right) r \frac{d r}{d t}=1 \quad \alpha=\frac{\mu \phi r_{p}}{2 \gamma \cos \theta_{p} \kappa}\left[s / m^{2}\right]
\end{aligned}
$$

with $\gamma[N / m]$ the surface tension, $\theta_{p}\left[^{\circ}\right]$ the contact angle, $\alpha$ the wicking constant [19] and $C_{1}\left[s / m^{4}\right]$ and $C_{2}\left[\mathrm{~s} / \mathrm{m}^{2}\right]$ the channel constants. A detailed derivation of Equation (5) is provided in Section A.2 of the Appendix. To fully represent the dynamic state of a SIMPLE chip over time, it is required to express the radius $r$ in function of time $t, r(t)$. However, the solution to (5) cannot be inverted in closed form because of the channel resistance terms $C_{1}\left(r^{2}-r_{0}^{2}\right)$ and $C_{2}$. To evaluate the importance of the channel resistance terms in Equation (5) the constants $C_{1}\left[\mathrm{~s} / \mathrm{m}^{4}\right]$ and $C_{2}\left[\mathrm{~s} / \mathrm{m}^{2}\right]$ are non dimensionalized with respect to $r_{0}$ and $\alpha$ yielding to $C_{1}^{*}=C_{1} r_{0}^{2} / \alpha[-]$ and $C_{2}^{*}=2 C_{2} / \alpha$ [-] (Section A.3 of the Appendix). Using the values given in Section 3.1 and typical design parameters $\left(w_{s}=\right.$ $0.5 \mathrm{~mm} ; w_{w}=3 \mathrm{~mm} ; h_{w}=h_{s}=0.153 \mathrm{~mm}$ ), the expected values of the non-dimensional channel constants $\left(C_{1}^{*}=5.3 \cdot 10^{-4}\right.$ and $\left.C_{2}^{*}=1.1 \cdot 10^{-2}\right)$ are not significant. Therefore, we neglect the channel terms in (1), assuming that the pumping mechanism is not dependent on the geometry of the WLC and SLC for the typical SIMPLE chip dimensions.

Simplifying (5), solving for $t(r)$ and inverting the result as elaborated in the Appendix (Section A.4), the radial imbibition Equation (6) is obtained, similar to the one described by Conrath et al. [19]. While, Equation (6) only describes the imbibition in the PM, similar to what happens in paper-based microfluidics systems, we further apply it to account for the transport of WL and SL. In this work, Equation (7) and (8) were derived for the first time, describing the volume of liquid in the WLC $\left(V_{w}(t)\right)$ and SLC $\left(V_{s}(t)\right)$ in function of time, respectively. 


$$
\begin{gathered}
r(t)=r_{0} \sqrt{\frac{e f(t)}{W(f(t))}} \text { with } f(t)=e^{-1}\left(\frac{4 t}{\alpha r_{0}^{2}}-1\right) \\
V_{w}(t)=w_{w} h_{w} \ell_{w}(t)=w_{w} h_{w} \ell_{0}-\phi \frac{\beta h_{p}}{2}\left(r^{2}(t)-r_{0}^{2}\right) \\
V_{s}(t)=w_{s} h_{s} \ell_{s}(t)=\phi \frac{\beta h_{p}}{2}\left(r^{2}(t)-r_{0}^{2}\right)
\end{gathered}
$$

Together, these three equations form the analytical model of the SIMPLE chip. From this model, we formulate the following hypotheses:

1. The imbibition equation is independent from the design parameters of the SIMPLE chip, except for the initial radius $r_{0}$

2. The channel (WLC or SLC) flow rate in the SIMPLE chip is directly dependent on the angle $(\beta)$ and height $\left(h_{p}\right)$ of the porous material.

3. The channel (WLC or SLC) flow rate in the SIMPLE chip is independent from the geometry of the channels $\left(w_{w}, w_{s}, h, \ell_{0}\right)$.

\subsection{Grey-box approach}

A common concern in paper-based microfluidics is the high variability of paper properties, such as the pore radius and porosity, which greatly affects the performance of paper-based chips. Since the SIMPLE chip integrates a paper pump, the effect of the paper variability was investigated and paper properties (i.e. pore radius and porosity) were defined as the normal distributions $\mathrm{A} \sim N\left(\bar{\alpha}, \sigma_{\alpha}^{2}\right)$ and $\Phi \sim N\left(\bar{\phi}, \sigma_{\phi}^{2}\right)$, respectively. Then, these distributions were substituted in Equations (6), (7) and (8) to obtain a statistical representation of the pumping behavior In practice, the model is used to individually estimate $\hat{\alpha}_{i}$ and $\hat{\phi}_{i}$ for $N$ sample chips $i$, which are then used to estimate the mean of A and $\Phi$ with a student-t distribution. To support the assumption of normally distributed paper properties, Shapiro-Wilk normality tests were carried out [28]. From these estimated distributions, the expected pumping dynamics are described as prediction intervals in function of time, as shown in Figure 2. These prediction intervals are estimated using the first-order Delta method, as described in the Appendix (Section A.5) [29]. Furthermore, by assuming the PM can be adequately described by a simple capillary bundle model, we reduced the expression of the wicking parameter to a function of the effective pore radius $\widehat{r_{p}}=\frac{4 \mu}{\gamma \alpha}[m]$ (Section B.3 of the Appendix). As they are easier to interpret, $\widehat{r_{p}}$ values are used to report the estimated wicking parameter. 


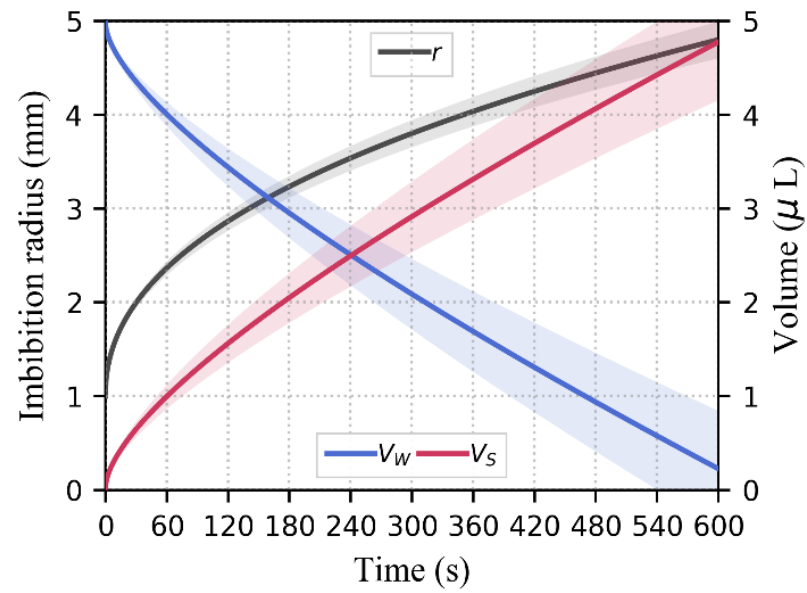

Figure 2: Analytical example of SIMPLE pumping dynamics with the curve of the imbibition radius $(\boldsymbol{r})$, the WLC curve $\left(\boldsymbol{V}_{\boldsymbol{W}}\right)$ and the SLC curve $\left(\boldsymbol{V}_{\boldsymbol{S}}\right)$ as given in Equations (6), (7) and (8)respectively. All curves are constructed from random distributions of $\mathbf{A}$ and $\mathbf{\Phi}$; expected values (lines) are calculated from the mean of the distributions while the prediction intervals (shaded area) are calculated by taking into account the covariance of $\mathbf{A}$ and $\mathbf{\Phi}$.

\section{Materials and Methods}

\subsection{Materials}

Double-sided pressure sensitive adhesive (PSA) tape (200MP 7956MP, 3M) and PVC transparency foils $(180 \mu \mathrm{m}$ thick) were utilized. Whatman filter paper grade 40 was purchased from The Merck group - Sigma Aldrich (Belgium). From the reported parameters of the Whatman 40 (i.e. thickness of $210 \mu \mathrm{m}$, particle retention of $8 \mu \mathrm{m}$, base weight of $92 \mathrm{~g} / \mathrm{m}^{2}$, Herzberg filtration time of $340 \mathrm{~s}$ ), the pore radius, porosity and permeability [19] of the paper were calculated in $4 \mu \mathrm{m}, 69.8 \%$, and 0.062 $\mu \mathrm{m}^{2}$, respectively.

\subsection{SIMPLE fabrication}

The microfluidic SIMPLE devices were fabricated according to the low-cost and rapid prototyping method presented in Yuen et al. [30] and Kokalj et al. [15]. In short, the microfluidic channels were cut in the PSA layer by using a digital tabletop craft cutter (Maxx Air 24", KNK, USA). Microfluidic channel height was determined by the PSA thickness, in this case $0.153 \mathrm{~mm}$. The PSA layer was sandwiched between bottom and top PVC layers, with the top layer featuring the necessary inlet, prefilling and vent holes. A PM, in this case filter paper (i.e. Whatman grade 40,), cut with a digital craft cutter (Cameo, Silhouette, USA), was inserted into its chamber during the assembly. Each SIMPLE chip was then prefilled with colored liquid. In particular blue and red food colors diluted in distilled water (1:100) were used as WL and SL respectively. As similarly reported in our previous work [15,26,27], liquids were pipetted into the respective chambers through the prefilling holes, which were then sealed with a PSA patch. All designs of the microfluidic chips used in this work with respective dimensions are presented in the Appendix (Section $\mathrm{C}$ ).

\subsection{Data acquisition and processing}

All experiments were recorded using a CMOS camera (HD Pro c920 webcam, Logitech, Switzerland) while all data processing was performed using Python [31]. Volume marks were cut into the PSA 
layers at predefined intervals next to the channels (every $1 \mu \mathrm{L}$ for the SLC and every $2 \mu \mathrm{L}$ for the WLC) and on the side of the PM chamber every $2 \mu \mathrm{L}$. The recorded videos were analyzed and the time at which the liquid front reached each mark was measured. In the PM, the measurement of the radius was approximated by looking at the timing of the imbibition front at three separate positions (left, middle and right) and averaging the results (see Section B.1 of the Appendix). This was eventually used to calculate the SLC flow rate of the SIMPLE chips as ratio between the SL volume displaced and the time needed. Moreover, the flow rate was calculated starting from 15 seconds after the SIMPLE activation until its termination to allow the stabilization of the flow rate which is substantially higher in the first few seconds due to the activation step and the intrinsic nature of the pumping behavior.

Due to the limitations of the fabrication method and the manual assembly of the chip, a small number of experiments deviated clearly from all others and were considered as outliers. To identify these outliers in a rigorous and systematic way, a two-sided t-test comparing the values of each individual observation to the distributions of the other observations at each measurement point was used (see Figure A.3 of the Appendix). Observations with highly significant $(p<0.01)$ and sustained deviations were excluded from the final data set. However, at least three repetitions were used for each experiment.

For all experiments, two types of curves were measured: i) the imbibition curve, which corresponds to the imbibition distance (radius, $r$ ) of the WL in the PM in function of time and ii) the channel transport curve, corresponding to the displaced volume of WL or SL in the respective channels in function of time. The relevant properties of Whatman 40 filter paper in a SIMPLE pump were estimated from these curves as detailed in the Appendix (Section B.2).

\section{Results and discussion}

\subsection{Effective pore radius and porosity estimation for enclosed porous materials}

As mentioned in the introduction, several groups investigated and modeled the imbibition of liquids in porous materials. However, in those cases the porous material (i.e. filter paper) was not enclosed in a plastic chip (contrary to the SIMPLE approach). The enclosing of the filter paper in a polymeric chip might affect the imbibition behavior, for instance due to compression of the paper during fabrication.

To estimate the properties of the Whatman 40 filter paper in the chip and to validate the proposed model, the imbibition of WL in paper circular sectors (central angles of $30^{\circ}, 60^{\circ}, 90^{\circ}$ ) was tested in SIMPLE chips made only of PM and WLC. The initial radius of the imbibition front $\left(\mathrm{r}_{0}\right)$ was kept constant at $1 \mathrm{~mm}$ since, as shown in the Appendix (Section D.1), varying this parameter has a strong influence on the paper imbibition and the flow rate in the channel. The imbibition radius and the WL volume absorption were monitored. As depicted in Figure $3 \mathrm{~A}$, the measurements of the imbibition radius (dots) allowed to estimate $\widehat{r_{p}}$ for all three angles tested $\left(30^{\circ}, 60^{\circ}, 90^{\circ} ; \mathrm{R}^{2}>0.99\right.$ for all observations). An average $\widehat{r_{p}}$ of $0.24 \pm 0.03 \mu \mathrm{m}$ was found, which is comparable to the $\widehat{r_{p}}$ value calculated $(0.18 \mu \mathrm{m})$ based on the properties of Whatman 40 filter paper (Section B.3 of the Appendix). The Shapiro-Wilk normality test yields p-values of 0.73 and 0.45 for the calculated distributions $\widehat{A}$ and $\widehat{\Phi}$ respectively, therefore we do not reject our assumption that $A$ and $\Phi$ are normally distributed. The resulting 95\% confidence interval of the analytical model (shaded area in Figure 3) corresponds well to the confidence interval of the experimental data (error bars in Figure 
3). Since the WL volume was kept constant for all angles, the imbibition radius reached at pump termination was different for the different angles, being smaller for wider angles and vice versa. Figure $3 \mathrm{~B}$ shows the volume of WL remained in the channel, due to absorption in the PM, over time for different paper angles. From these data, the effective porosity of the paper $(\hat{\phi})$ was estimated to be $0.52 \pm 0.03$. As for the imbibition graph, the experimental points were overlaid with the analytical model curves derived from the estimated $\widehat{r_{p}}$ and $\hat{\phi}$. For both graphs, the experimental results were in line with both the analytical model presented here and the previous work by Kokalj et al. [15], confirming the influence of the filter paper shape on the WL flow rate in the channel. To evaluate the dependence of $\widehat{r_{p}}$ and $\hat{\phi}$ from the $\beta$, the statistical significance of their slope in function of $\beta$ was evaluated with an OLS regression, yielding p-values of 0.67 and 0.16 , and therefore confirming the first two hypotheses proposed in Section 2.2.
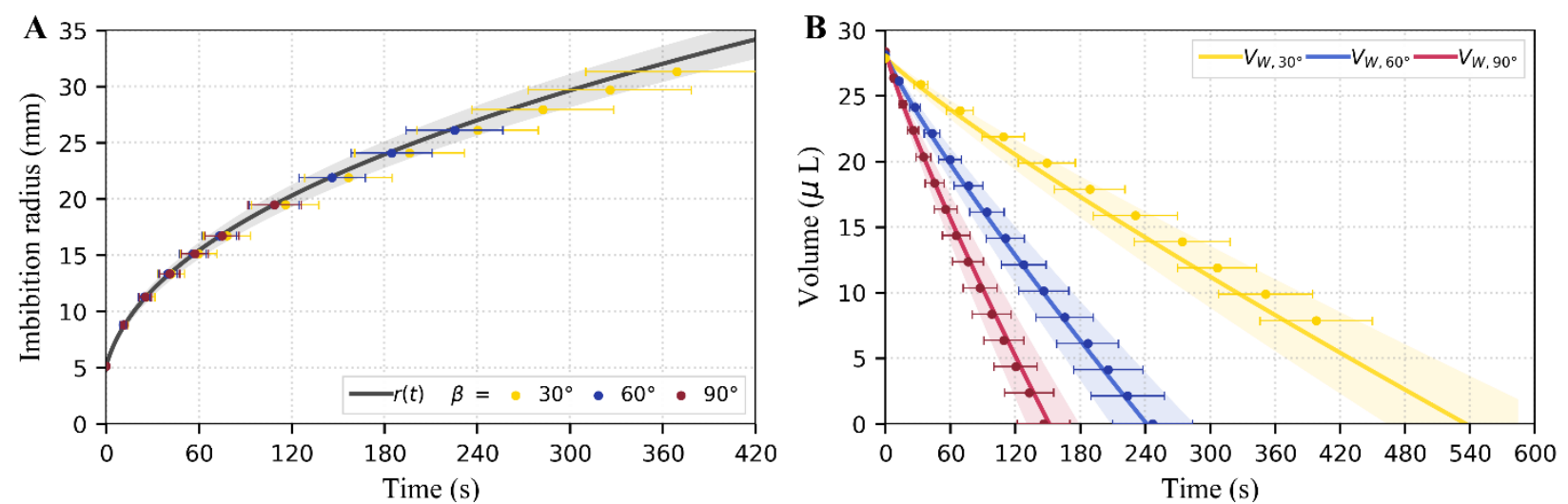

Figure 3: Characterization of Whatman 40 filter paper for different shapes $\left(30^{\circ}, 60^{\circ}, 90^{\circ}\right.$ circular sector) in a SIMPLE chip without SLC. A) Radial imbibition measurements (dots) in the PM for the three different angles compared to the fitted curve (solid grey line) and the predicted region (shaded area) based on the analytical model. B) Volume of WL left in the WLC in function of time for the three different angles. Solid lines and shaded area correspond to the fitted curve and prediction region based on the analytical model. For both graphs, the average data (at least 4 repetitions per experiment) are displayed as dots and error bars represent their $95 \%$ confidence interval.

4.2. Model validation for SIMPLE platform

Following the model description in section 2 and the paper characterization in terms of effective pore radius and effective porosity in Section 4.1, the developed model was validated on a complete SIMPLE pump. The influence of different chip parameters as i) PM angle, ii) SLC width and iii) PM volume for a given angle, on the SL flow rate was investigated.

\subsubsection{Influence of porous material angles on sample liquid flow rate} As confirmed in Section 4.1, the PM shape, in this case circular sectors of different angles, had a strong influence on the absorption rate of the WL. However, for POC applications, it is more important to control and tune the flow rate of the SL instead. For this reason, the effect of different porous material angles on the SL flow rate was investigated here. The designs of the chips (for details see Section C of the Appendix), were kept the same as the ones used in Section 4.1, except for the addition of an SLC (width of $0.6 \mathrm{~mm}$ ). Both the imbibition radius and the SL volume displaced in function of time were monitored (Figure 4) and compared to predictions based on the $\widehat{r}_{p}$ and $\hat{\phi}$ values reported in Section 
4.1. As expected, the measured imbibition radius values fell inside the prediction region (Figure $4 \mathrm{~A}$ ). Most importantly, as shown in Figure $4 \mathrm{~B}$, the volume of SL pulled into the channel over time was strictly dependent on the PM angle, following the rule that wider angles lead to faster flow rates and vice versa. In particular, the average SL flow rate for SIMPLE chips with $30^{\circ}, 60^{\circ}, 90^{\circ} \mathrm{PM}$ was $3.6 \pm$ $0.1,6.4 \pm 1.5,11.3 \pm 2.4 \mu \mathrm{L} / \mathrm{min}$, respectively. More detailed explanation on the average flow rare calculation and relative variation is given in Section B.4 of the Appendix.
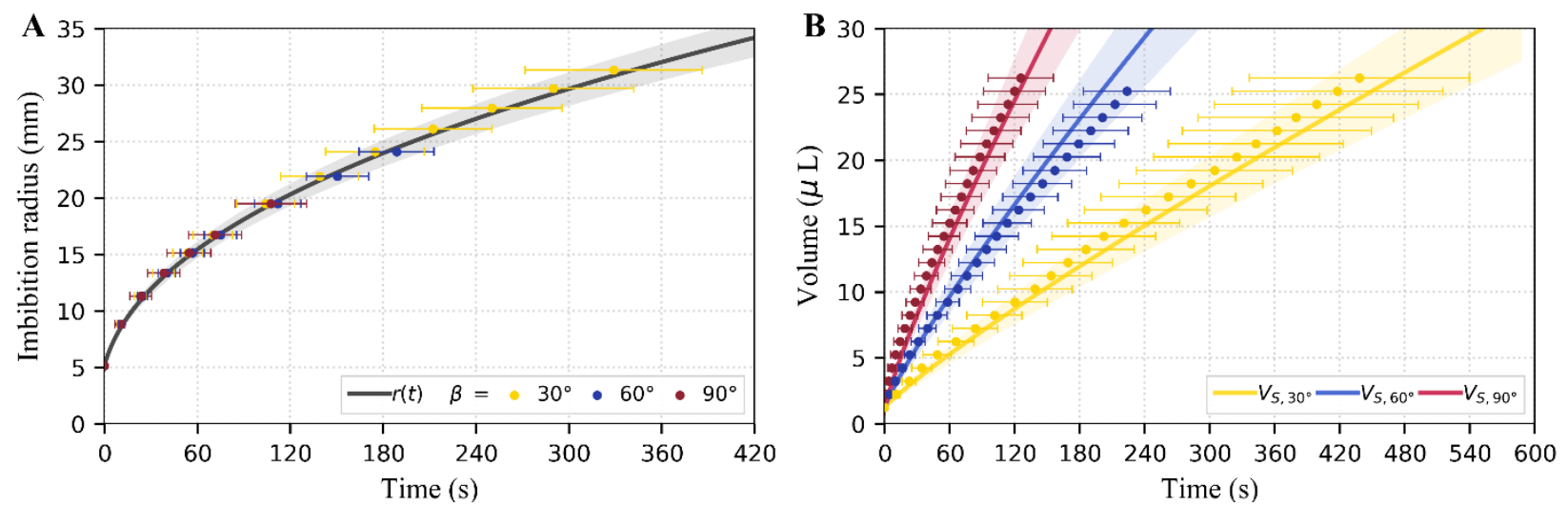

Figure 4: A) Measured radial imbibition of complete SIMPLE chips for three different porous material angles $\left(30^{\circ}, 60^{\circ}\right.$, $90^{\circ}$ ). B) Volume of sample liquid pulled during SIMPLE operation for different paper angles. For both graphs, the measured data are represented by dots and error bars represent their $95 \%$ confidence interval (at least 3 repetitions per experiment), while the average fitted model curve is shown as a solid line with its prediction region shaded.

The effective pore radius and porosity were calculated here as well, resulting in $0.24 \pm 0.04$ $\mu \mathrm{m}$ and $0.52 \pm 0.03$, respectively. These values are not significantly different ( $\mathrm{p}$-values of 0.90 and 0.85 respectively) from the ones obtained for the SIMPLE chips without SLC proving once more the independence of the estimated paper properties from the chip design and confirming the robustness of the model.

\subsubsection{Influence of sample channel width on sample liquid flow rate}

From the analytical derivation presented in Section 2, it was hypothesized that the influence of the SLC design (i.e. channel resistance) on the SL flow rate was negligible in case of low filter paper permeability and relatively low resistance channels. Channel resistance can be tuned by changing the channel surface or the channel geometry, with the latter used in this work. In a SIMPLE chip, due to the fabrication method and material used, the height of the channel was fixed by the height of the PSA layer (i.e. $0.153 \mathrm{~mm}$ ) while the minimum width was limited by the cutter resolution $(0.5 \mathrm{~mm})$. To demonstrate the independence of the SL flow rate from channel resistance, SIMPLE chips were designed with three different widths of the SLC, namely $0.6 \mathrm{~mm}$ or high resistance (same as in Section 4.2.1), $1 \mathrm{~mm}$ or medium resistance and $1.5 \mathrm{~mm}$ or low resistance, for three different angles of the PM (i.e. $30^{\circ}, 60^{\circ}, 90^{\circ}$ ). Figure 5 depicts the absolute time difference needed to pull a defined amount of sample liquid (steps of $1 \mu \mathrm{L}$ ) between low and medium resistance chips (light red dots), medium and high resistance chips (red dots) and high and low resistance chips (dark red dots) for a PM of $90^{\circ}$ angle. As can be seen, the absolute average time difference was close to $0 \mathrm{~s}$ in all cases proving that the SLC dimension has no major effect on the SL flow rate and confirming the third hypothesis presented in Section 2.2. As reference, the average SL flow rate for low, medium and high channel 
resistance was measured in $11.9 \pm 1.5,12.3 \pm 3.3,11.3 \pm 2.4 \mu \mathrm{L} / \mathrm{min}$, respectively. As reference, 118.0 $\pm 9.0,113.5 \pm 23.1,117.1 \pm 14.4$ s were needed on average to pull $25 \mu \mathrm{L}$ of SL for low, medium and high resistance chip, respectively.

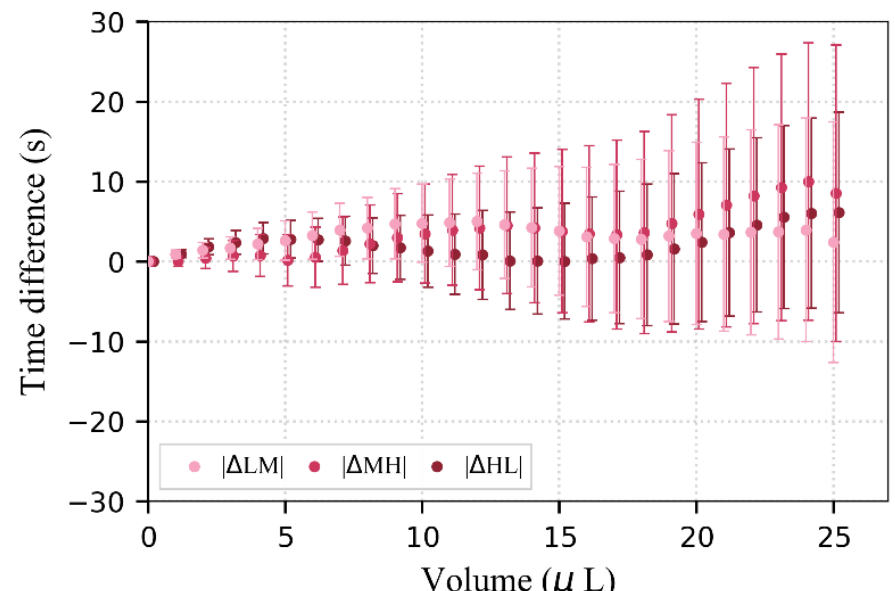

299

300

301

302

303

304

305

306

307

308

309

310

311

312

313

314

315

316

317

318

319

320

321

322

323

324

325

326

327

Figure 5: Effect of SLC resistance on SL flow rate. Dots represent the absolute value of the difference of the time needed to pull $1 \mu \mathrm{L}$ of SL between SIMPLE chips (with $90^{\circ} \mathrm{PM}$ ) with different channel resistance. In particular, light red dots represent the average difference between low and medium resistance chips, red dots between medium and high resistance chips, and dark red dots between high and low resistance chips. At least 3 repetitions per experiment were performed and error bars represent the $95 \%$ confidence interval.

\subsubsection{Influence of porous material volume on sample liquid flow rate}

In order to fully validate the SIMPLE analytical model, the influence of the PM volume, for a specific filter paper and fixed circular sector angle, on the SL flow rate was investigated. According to the model hypothesis, the volume of the filter paper should not have any influence on the SL flow rate and in order to assess this, three different designs with the same paper type and angle (i.e. $60^{\circ}$ ) but different targeted pumping volumes (i.e. 15, 30, $45 \mu \mathrm{L}$ of SL) were tested. As depicted in Figure 6, all the experimental values recorded over time laid within the prediction region (shaded area) and follow closely the fitted curve (solid line). In particular, a flow rate of $7.5 \pm 1.1,6.8 \pm 1.4,7.9 \pm 1.3$ $\mu \mathrm{L} / \mathrm{min}$ was calculated for $60^{\circ}$ SIMPLE chips with pumping volumes of $15,30,45 \mu \mathrm{L}$, respectively. The flow rate was calculated only in the overlapping region of the curves (i.e. until termination of the $15 \mu \mathrm{L}$ SIMPLE chip), since the smaller pump stopped before the others. These results confirmed the absence of dependence between the SIMPLE pumping volume and the corresponding SL flow rate, as expected from the model discussed in Section 2.

Additionally, Figure 6 contains information on the pumping efficiency of the SIMPLE platform. In fact, the chips were designed to pull 15,30 or $45 \mu \mathrm{L}$ of SL into the channel, corresponding to the volume of WL prefilled in the chip. Since the pump was terminated when all the WL was absorbed in the PM, it was expected that the same amount of SL was drawn into the network. However, the SL volume pulled into the chip was slightly lower resulting in an efficiency of 90.2, 89.5, $87.5 \%$ for SIMPLE chips targeting 15, 30 or $45 \mu \mathrm{L}$, respectively. While this decreased efficiency cannot be explained by our current model, it should not impact the predictive capability of our model. In practice, it will only result in a discrepancy between the WLC- and SLC-based estimates of $\hat{\phi}$, which we can take into account during the design process. However, the downwards trend suggests that 
the pumping efficiency may vary from design to design, which is an important factor to consider in further research.

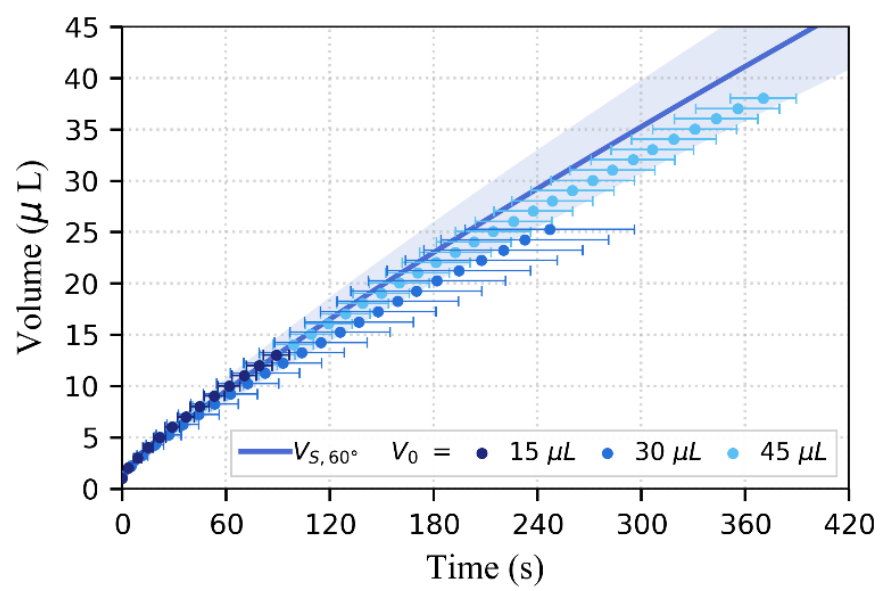

Figure 6: Effect of the PM volume on SL flow rate. Average volumes (at least 4 repetitions per experiment) of SL pumped over time for SIMPLE chips with $60^{\circ}$ circular sector PM but targeting different pumping volume, namely $15 \mu \mathrm{L}$ (dark blue dots), $30 \mu \mathrm{L}$ (blue dots) and $45 \mu \mathrm{L}$ (light blue dots). The average fitted model curve is shown as a solid blue line with its prediction region shaded. Error bars represent the $95 \%$ confidence interval.

\subsection{Model as design tool to achieve targeted sample liquid flow rate}

In the previous sections, the analytical model was validated under different conditions and consistently showed a good agreement with the measured data. However, the main goal of this work was to use this analytical model as a smart design tool which should take as input the desired SL flow rate and provide as output the specific PM angle to obtain such flow rate on a SIMPLE chip.

For this reason, the model was used in combination with the paper parameters $\widehat{r}_{p}$ and $\hat{\phi}$ estimated in Section 4.1, to find the correct PM angles in order to obtain three specific SL flow rates, namely $1.5,5,15 \mu \mathrm{L} / \mathrm{min}$, which cover a wide flow rate range for microfluidic applications. According to our model, the PM angles needed to provide the aforementioned flow rates were $15^{\circ}, 42.5^{\circ}$, and $112.5^{\circ}$, respectively (Section B.4 of the Appendix). As shown in Figure 7, the measured SL volume pumped over time followed closely the mean curve (solid lines) and fell inside the prediction region (shaded area) for all three flow rates targeted. In particular, an SL flow rate of $1.5 \pm 0.3,5.3 \pm 1.5,15.2$ $\pm 2.7 \mu \mathrm{L} / \mathrm{min}$ was measured for $15^{\circ}, 42.5^{\circ}$, and $112.5^{\circ} \mathrm{PM}$ angle, respectively. These values are close to the targeted flow rates, proving the prediction capability of the developed model which can be used to design specific SIMPLE chips according to application requirements (i.e. flow rate, pumping volume, channel width) without time-consuming and empirical design optimization iterations. 


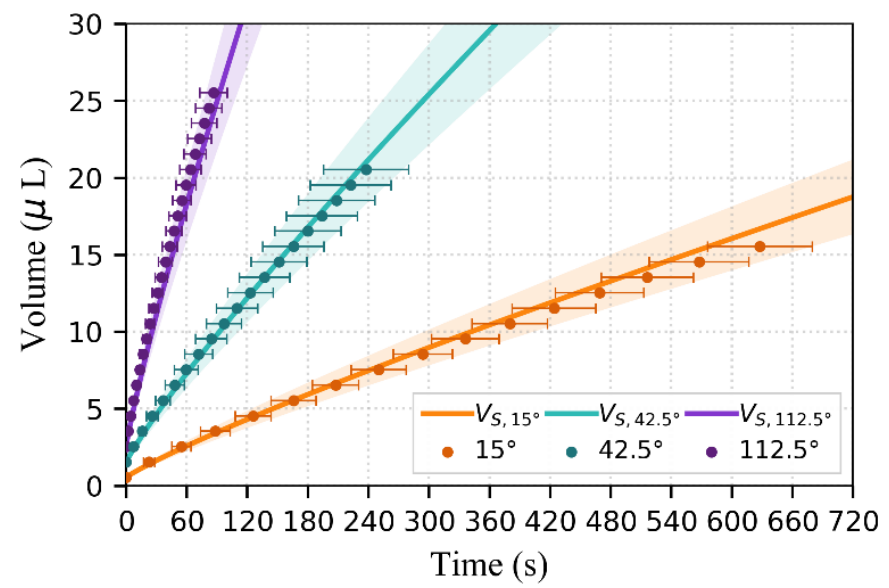

Figure 7: Volume of SL pulled during SIMPLE operation for different paper angles (i.e. $15^{\circ}, 42.5^{\circ}, 112.5^{\circ}$ ). These angles resulted in $1.5 \pm 0.3,5.3 \pm 1.5,15.2 \pm 2.7 \mu \mathrm{L} / \mathrm{min}$ SL flow rate, close to the ones predicted by the analytical model. Measured data are represented by dots and error bars represent their $95 \%$ confidence interval (at least 4 repetitions per experiment), while the average fitted model curve is shown as a solid line with its prediction region shaded.

\section{Conclusions}

Starting from models of radial capillary imbibition into porous materials, an analytical model of the pumping dynamics of the SIMPLE platform was developed. Taking into account the scaling of geometrical parameters relative to the paper properties, it was hypothesized that the SL flow rate was dependent on the PM shape but independent from the PM volume and channel geometry. Then, the PM (i.e. filter paper) properties (i.e. effective pore radius and effective porosity) were fitted from observations of a chip made only of a WL channel and PM. Their distributions were used to predict SIMPLE pumping behavior for a multitude of different designs. Using these predictions, the model was validated experimentally for different PM angles (i.e. $30^{\circ}, 60^{\circ}, 90^{\circ}$ ), PM volumes (i.e. $15,30,45$ $\mu \mathrm{L}$ ), and SLC width (i.e. $0.6,1,1.5 \mathrm{~mm}$ ) corresponding to high, medium and low resistance. The experimental results were in great agreement with the model, confirming all the hypotheses made. Finally, the model was used as smart tool to design SIMPLE chips working at predefined flow rates resulting in chips achieving $1.5 \pm 0.3,5.3 \pm 1.5,15.2 \pm 2.7 \mu \mathrm{L} / \mathrm{min}$, matching the targeted flow rates (i.e. $1.5,5,15 \mu \mathrm{L} / \mathrm{min}$ ).

Overall, it can be concluded that the analytical approach and the hypotheses proposed in this work are a useful tool for the design of microfluidic chips based on the SIMPLE concept. From a basic set of equations and a small set of initial measurements, the expected dynamics of the channel flow rate of a SIMPLE chip with unknown paper properties can be predicted, giving more insight into the pumping process than the previous assumption of constant flow rate [15]. This is crucial in implementing the SIMPLE concept in more complex LOC devices for POC applications, since knowledge of the characteristics and limitations of the pump is important to achieve an optimal chip design.

\section{Acknowledgments}

The research leading to these results has received funding from the Research Foundation - Flanders (FWO SB 1S65018N), and the KU Leuven (C3 project 3E170458, C2 project C24/16/022). 


\section{Abbreviations and symbols}

\begin{tabular}{|c|c|c|}
\hline 382 & SLC & sample liquid channel \\
\hline 383 & WLC & working liquid channel \\
\hline 384 & WL & working liquid \\
\hline 385 & SL & sample liquid \\
\hline 386 & PM & porous material \\
\hline 387 & $\gamma$ & surface tension (water) \\
\hline 388 & $\mu$ & dynamic viscosity (water) \\
\hline 389 & $\theta_{p}$ & contanct angle (water) \\
\hline 390 & $r_{p}$ & pore radius \\
\hline 391 & $\widehat{r_{p}}$ & effective pore radius \\
\hline 392 & $\phi$ & porosity \\
\hline 393 & $\hat{\phi}$ & effective porosity \\
\hline 394 & $\Phi$ & porosity distribution \\
\hline 395 & $\kappa$ & permeability (water) \\
\hline 396 & $\alpha$ & wicking constant \\
\hline 397 & A & wicking constant distribution \\
\hline 398 & $w_{w}$ & WLC width \\
\hline 399 & $w_{S}$ & SLC width \\
\hline 400 & $w_{0}$ & initial width (PM) \\
\hline 401 & $h$ & channel height \\
\hline 402 & $h_{w}$ & WLC height \\
\hline 403 & $h_{s}$ & SLC height \\
\hline 404 & $h_{p}$ & PM height \\
\hline 405 & $\ell_{w}$ & WLC filled length \\
\hline 406 & $\ell_{s}$ & SLC filled length \\
\hline 407 & $\ell_{0}$ & initial WLC filled length \\
\hline 408 & $r$ & imbibition front radius \\
\hline 409 & $r_{0}$ & initial imbibition front radius \\
\hline 410 & $t$ & time \\
\hline 411 & $\beta$ & central angle of the PM \\
\hline 412 & $A_{w}$ & WLC cross-section \\
\hline 413 & $A_{s}$ & SLC cross-section \\
\hline 414 & $V_{w}$ & WLC filled volume \\
\hline 415 & $V_{s}$ & SLC filled volume \\
\hline 416 & $R_{w}$ & WLC hydraulic resistance \\
\hline 417 & $R_{s}$ & SLC hydraulic resistance \\
\hline 418 & $R_{w}^{\prime}$ & per-length WLC hydraulic resistance \\
\hline 419 & $R_{s}^{\prime}$ & per-length SLC hydraulic resistance \\
\hline 420 & $C_{1}$ & dynamic channel constant \\
\hline 421 & $C_{2}$ & static channel constant \\
\hline
\end{tabular}




\section{References}

423 [1] G. Comina, A. Suska, D. Filippini, Towards autonomous lab-on-a-chip devices for cell phone

[2] M. Zimmermann, H. Schmid, P. Hunziker, E. Delamarche, Capillary pumps for autonomous capillary systems., Lab Chip. 7 (2007) 119-25. doi:10.1039/b609813d.

[3] W. Guo, J. Hansson, W. van der Wijngaart, Capillary Pumping Independent of Liquid Sample Viscosity, Langmuir. 32 (2016) 12650-12655. doi:10.1021/acs.langmuir.6b03488.

[4] L. Gervais, E. Delamarche, Toward one-step point-of-care immunodiagnostics using capillarydriven microfluidics and PDMS substrates., Lab Chip. 9 (2009) 3330-7. doi:10.1039/b906523g.

[5] E.-C. Yeh, C.-C. Fu, L. Hu, R. Thakur, J. Feng, L.P. Lee, Self-powered integrated microfluidic pointof-care low-cost enabling (SIMPLE) chip, Sci. Adv. 3 (2017) e1501645. doi:10.1126/sciadv.1501645.

[6] P. Novo, V. Chu, J.P. Conde, Integrated optical detection of autonomous capillary microfluidic immunoassays:a hand-held point-of-care prototype, Biosens. Bioelectron. 57 (2014) 284-291. doi:10.1016/J.BIOS.2014.02.009.

[7] E. Fu, T. Liang, P. Spicar-Mihalic, J. Houghtaling, S. Ramachandran, P. Yager, Two-dimensional paper network format that enables simple multistep assays for use in low-resource settings in the context of malaria antigen detection, Anal. Chem. 84 (2012) 4574-4579. doi:10.1021/ac300689s.

[8] A.W. Martinez, S.T. Phillips, G.M. Whitesides, E. Carrilho, Diagnostics for the developing world: microfluidic paper-based analytical devices., Anal. Chem. 82 (2010) 3-10. doi:10.1021/ac9013989.

[9] R. Safavieh, D. Juncker, Capillarics: pre-programmed, self-powered microfluidic circuits built from capillary elements, Lab Chip. 13 (2013) 4180. doi:10.1039/c3lc50691f.

[10] T. Horiuchi, K. Hayashi, M. Seyama, S. Inoue, E. Tamechika, Cooperative Suction by Vertical Capillary Array Pump for Controlling Flow Profiles of Microfluidic Sensor Chips, Sensors. 12 (2012) 14053-14067. doi:10.3390/s121014053.

[11] R. Safavieh, A. Tamayol, D. Juncker, Serpentine and leading-edge capillary pumps for microfluidic capillary systems, Microfluid. Nanofluidics. 18 (2015) 357-366. doi:10.1007/s10404-014-1454-3.

[12] B. Chumo, M. Muluneh, D. Issadore, Laser micromachined hybrid open/paper microfluidic chips, Biomicrofluidics. 7 (2013) 064109. doi:10.1063/1.4840575.

[13] J. Wang, H. Ahmad, C. Ma, Q. Shi, O. Vermesh, U. Vermesh, J. Heath, A self-powered, one-step chip for rapid, quantitative and multiplexed detection of proteins from pinpricks of whole blood, Lab Chip. 10 (2010) 3157-62. doi:10.1039/c0lc00132e.

[14] F. He, J. Grimes, S.D. Alcaine, S.R. Nugen, A hybrid paper and microfluidic chip with electrowetting valves and colorimetric detection, Analyst. 139 (2014) 3002. doi:10.1039/c3an01516e.

[15] T. Kokalj, Y. Park, M. Vencelj, M. Jenko, L.P. Lee, Self-powered Imbibing Microfluidic Pump by Liquid Encapsulation: SIMPLE., Lab Chip. 14 (2014) 4329-33. doi:10.1039/c4lc00920g.

[16] S.-J. Kim, S. Paczesny, S. Takayama, K. Kurabayashi, Preprogrammed capillarity to passively control system-level sequential and parallel microfluidic flows, Lab Chip. 13 (2013) 2091. doi:10.1039/c3lc50187f.

[17] A. Böhm, F. Carstens, C. Trieb, S. Schabel, M. Biesalski, Engineering microfluidic papers: effect of fiber source and paper sheet properties on capillary-driven fluid flow, Microfluid. Nanofluidics. 16 (2014) 789-799. doi:10.1007/s10404-013-1324-4. 
[18] E. Evans, E.F.M. Gabriel, W.K.T. Coltro, C.D. Garcia, Rational selection of substrates to improve color intensity and uniformity on microfluidic paper-based analytical devices, Analyst. 139 (2014) 2127-2132. doi:10.1039/C4AN00230J.

[19] M. Conrath, N. Fries, M. Zhang, M.E. Dreyer, Radial Capillary Transport from an Infinite Reservoir, Transp. Porous Media. 84 (2010) 109-132. doi:10.1007/s11242-009-9488-9.

[20] E. Fu, S.A. Ramsey, P. Kauffman, B. Lutz, P. Yager, Transport in two-dimensional paper networks., Microfluid. Nanofluidics. 10 (2011) 29-35. doi:10.1007/s10404-010-0643-y.

[21] S. Mendez, E.M. Fenton, G.R. Gallegos, D.N. Petsev, S.S. Sibbett, H.A. Stone, Y. Zhang, G.P. López, Imbibition in Porous Membranes of Complex Shape: Quasi-stationary Flow in Thin Rectangular Segments, Langmuir. 26 (2010) 1380-1385. doi:10.1021/la902470b.

[22] X. Wang, J.A. Hagen, I. Papautsky, Paper pump for passive and programmable transport, Biomicrofluidics. 7 (2013) 14107. doi:10.1063/1.4790819.

[23] D. Shou, L. Ye, J. Fan, K. Fu, M. Mei, H. Wang, Q. Chen, Geometry-Induced Asymmetric Capillary Flow, Langmuir. 30 (2014) 5448-5454. doi:10.1021/la500479e.

[24] E. Elizalde, R. Urteaga, R.R. Koropecki, C.L.A. Berli, Inverse Problem of Capillary Filling, Phys. Rev. Lett. 112 (2014) 134502. doi:10.1103/PhysRevLett.112.134502.

[25] E. Elizalde, R. Urteaga, C.L.A. Berli, Rational design of capillary-driven flows for paper-based microfluidics., Lab Chip. 15 (2015) 2173-80. doi:10.1039/c4lc01487a.

[26] F. Dal Dosso, D. Decrop, E. Pérez-Ruiz, D. Daems, H. Agten, O. Al-Ghezi, O. Bollen, J. Breukers, F. De Rop, M. Katsafadou, J. Lepoudre, L. Lyu, P. Piron, R. Saesen, S. Sels, R. Soenen, E. Staljanssens, J. Taraporewalla, T. Kokalj, D. Spasic, J. Lammertyn, Creasensor: SIMPLE technology for creatinine detection in plasma, Anal. Chim. Acta. (2017). doi:10.1016/j.aca.2017.11.026.

[27] F. Dal Dosso, T. Kokalj, J. Belotserkovsky, D. Spasic, J. Lammertyn, Self-powered infusion microfluidic pump for ex vivo drug delivery, Biomed. Microdevices. 20 (2018) 44. doi:10.1007/s10544-018-0289-1.

[28] S.S. Shapiro, M.B. Wilk, An Analysis of Variance Test for Normality (Complete Samples), Biometrika. 52 (1965) 591. doi:10.2307/2333709.

[29] A. Ruckstuhl, Introduction to Nonlinear Regression, ZHAW Zürcher Hochschule Für Angew. Wissenschaften. 1 (2010). doi:10.1007/978-3-319-33455-4.

[30] P.K. Yuen, V.N. Goral, Low-cost rapid prototyping of flexible microfluidic devices using a desktop digital craft cutter., Lab Chip. 10 (2010) 384-7. doi:10.1039/b918089c.

[31] G. Van Rossum, F.L. Drake, The Python Language Reference Manual, Network Theory Ltd, 2011. https://dl.acm.org/citation.cfm?id=2011965 (accessed March 7, 2018). 